\title{
An unusual case of pustulotic arthro-osteitis affecting the leg, and erosive polyarthritis
}

\author{
Stuart H Ralston, P D R Scott, Roger D Sturrock
}

\begin{abstract}
Pustulotic arthro-osteitis is a syndrome, originally described in Japan, in which a sterile inflammatory osteitis of the sternoclavicular region occurs in combination with a non-erosive arthritis, sacroiliitis, and a pustulotic rash on the palms and soles. Although the disease has been reported in white populations, it is rarely seen by rheumatologists in the United Kingdom. The case of a Scottish woman is described in whom the diagnosis of pustulotic arthro-osteitis was overlooked because of an atypical site of the disease-the fibula, an erosive arthritis, and absence of a pustular rash at the time of her presentation with musculoskeletal symptoms. This case shows that the syndrome may easily by missed unless clinical suspicion is high and should be considered in any patient with culture negative osteitis, even outside the sternoclavicular region.
\end{abstract}

Pustulotic arthro-osteitis is a rheumatic syndrome of unknown cause, characterised by an inflammatory osteitis of the sternocostoclavicular region and palmoplantar pustulosis. ${ }^{1}$ In some cases a non-erosive oligoarthritis may occur, as may sacroiliitis and syndesmophyte formation. ${ }^{1}$ Because of these last abnormalities it has been proposed that the disease should be classified as a member of the seronegative spondyloarthritis group, though affected patients are seldom positive for HLA-B27. ${ }^{2}$ Hitherto, most reports of the disease have originated from Japan, ${ }^{2-4}$ and although the syndrome has also been described in white populations, ${ }^{5-9}$ it is rare in the United Kingdom. ${ }^{10}$

We now-describe a British patient who presented with an unusual form of arthroosteitis and palmoplantar pustulosis, in that the predominant site of the disease was the fibula and the arthritis was erosive.

The Centre for

Rheumatic Diseases, Glasgow Royal Infirmary, Glasgow G4 OSF $S$ H Ralston

R D Sturrock

Orthopaedic

Department,

Victoria Infirmary,

Glaseow

P D R Scott

Correspondence to: Dr Stuart H Ralston, Rheumatic Diseases Unit, Northern General Hospital, Edinburgh EHS 2DQ.

Accepted for publication 11 September 1989

\section{Case report}

A 60 year old woman presented in March 1984 with a three month history of pain and stiffness affecting the small joints of the hands, wrists, knees, and left leg. Five years previously she had attended a dermatologist because of a pustular rash affecting the palms of both hands and the soles of both feet but had not mentioned this at the time of her presentation with joint symptoms. Systematic inquiry was unremarkable apart from the fact that she had had mild Raynaud's syndrome for many years.

On physical examination she was found to have a symmetrical arthritis affecting the metacarpophalangeal and proximal interphalangeal joints of the hands, both wrists, and both knees. Although she had clinical evidence of mild sclerodactyly, there was no macroscopic abnormality of the nailfold vessels, or other clinical evidence of connective tissue disease. Physical examination was otherwise unremarkable apart from bony swelling of the lower part of the left fibula.

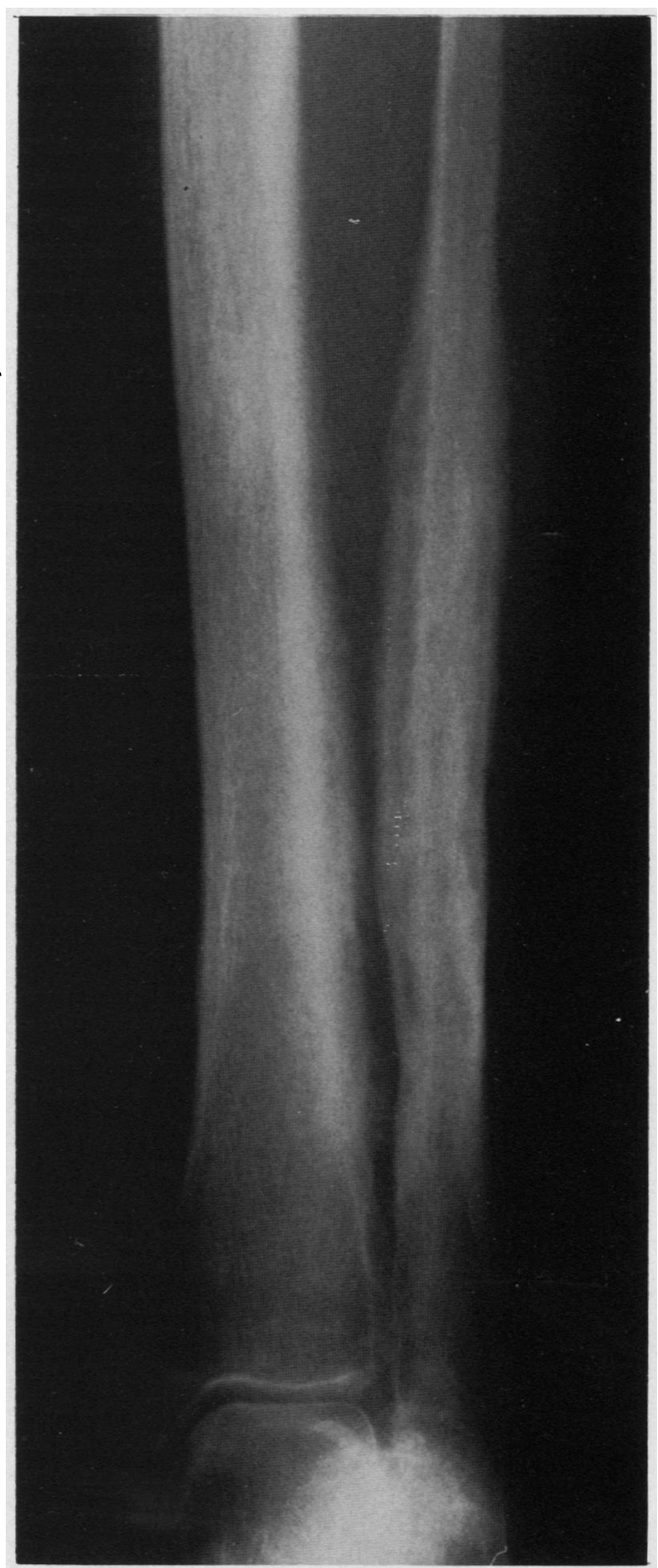

Figure 1: Radiograph of the leg at presentation showing enlargement and periostitis of left fibula and periostitis of left tibia. 
Biochemical and haematological screens were normal, erythrocyte sedimentation rate (ESR) was $15 \mathrm{~mm}$ in the first hour, and rheumatoid and antinuclear factors were negative. Although hand radiographs were normal, $x$ rays of the left leg showed sclerosis and expansion affecting the lower third of the left fibula (fig 1). An open biopsy showed mild chronic inflammatory changes and osteosclerosis. The appearances were consistent with a low grade osteitis, though cultures of material taken at operation were negative.

The patient was thought to have a combination of mild seronegative inflammatory arthritis and a low grade osteitis of the fibula. Treatment was started with flucloxacillin, analgesics, and nonsteroidal anti-inflammatory drugs, but she continued to experience pain in the left leg. Three years later she was referred to the centre for rheumatic diseases after a further episode of palmoplantar pustulosis and a worsening in the left leg pain. Clinical examination was essentially unchanged from before, except for the evidence of healing pustular lesions in both palms. Investigations were as follows: haemoglobin 133 g/l, white cell count $9.6 \times 10^{6} / 1$ with normal differential, platelets $359 \times 10^{6} / 1$, ESR $86 \mathrm{~mm} / \mathrm{h}$. Rheumatoid factor, antinuclear factor, and anti Ro, La, Sm, RNP, and anticentromere antibodies were negative. HLA typing showed the following results: HLA-A25; B5, 18; Bw4, 6 . Biochemical screening showed no abnormality apart from an increase in total globulins $37 \mathrm{~g} / 1$ (normal 23-33), though immunoglobulin subfractions IgA, IgG, and IgM were normal.

A bone scan showed 'hot spots' in the small joints of the hands, wrists, and both knees consistent with an inflammatory arthropathy. There were, in addition, abnormal focal

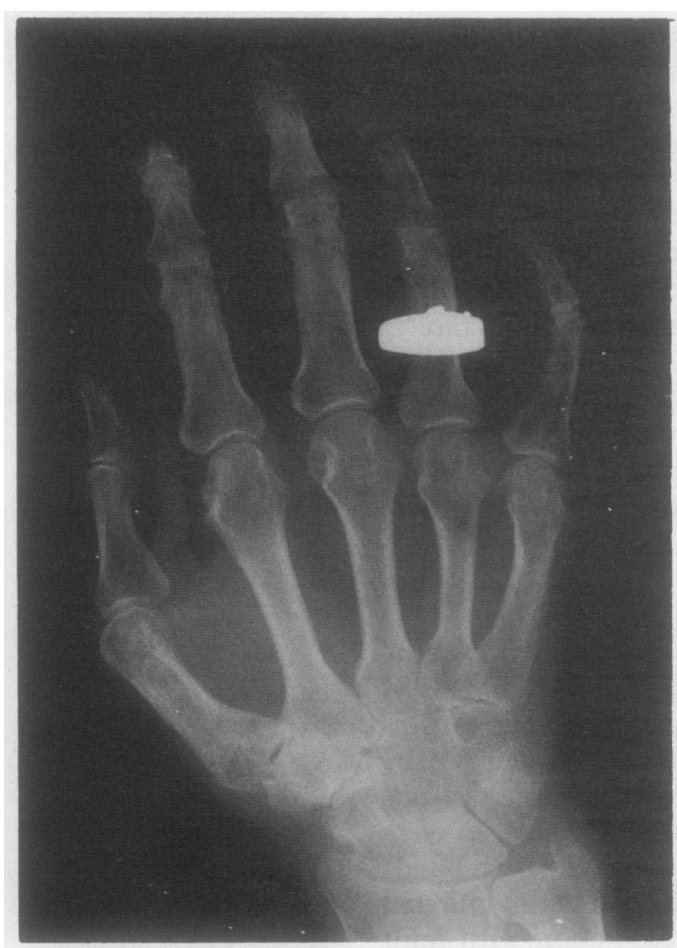

Figure 2: Radiograph of the left hand five years after presentation showing evidence of cartilage loss in the carpus, and non-proliferative erosions affecting the carpus and several of the metacarpophalangeal and proximal interphalangeal joints. The right hand was affected similarly. increases in tracer uptake at the lower ends of the left fibula and tibia, the anterior ends of the seventh and eighth ribs, and the posterior end of the third rib. An $x$ ray examination of the chest, pelvis, and lumbar spine showed no abnormality, but $x$ rays of the hands showed evidence of a symmetrical erosive arthritis affecting both wrists and several of the metacarpophalangeal and proximal interphalangeal joints in both hands (fig 2).

In view of the clinical features a diagnosis of arthro-osteitis and palmoplantar pustulosis was suspected and the antibiotics were stopped, with no significant deterioration in symptoms. Although the arthropathy is currently well controlled by non-steroidal anti-inflammatory drugs, she continues to experience persistent leg pain and because of this has been treated with local transcutaneous electrical nerve stimulation, with some improvement.

\section{Discussion}

The osteitis and hyperostosis have been confined to the sternocostoclavicular region in almost all previous reported cases of this syndrome. ${ }^{1-9}$ Although our patient almost certainly had osteitis affecting the ribs, as shown by the bone scan abnormalities of the thoracic cage, ${ }^{2}$ these lesions were asymptomatic and were not associated with any obvious radiological change. In contrast, there was marked expansion and periostitis of the left fibula, which was the major symptomatic problem throughout the patient's follow up. Despite the unusual distribution of lesions the diagnosis was almost certainly a variant of arthro-osteitis because of the characteristic histological and radiological changes, ${ }^{2-9}$ the association with palmoplantar pustulosis, 35 and the seronegative arthritis. ${ }^{2}$ The hyperglobulinaemia and raised ESR, which were the only significant laboratory abnormalities in our patient, have previously been noted by other workers in patients without obvious arthritis. ${ }^{8}$ Serum protein electrophoresis in such cases generally shows a non-specific pattern of increases in $\alpha_{2}$ or $\beta$ globulins, or both, ${ }^{8}$ but electrophoresis was not performed in our patient. The cause of these abnormalities, like the cause of the underlying disease process, remains unclear; unlike Edlund et al, who recently cultured Propionibacterium acnes in material from about $50 \%$ of patients with this syndrome, ${ }^{5}$ no organisms were cultured from samples taken at open biopsy in our patient. This agrees with the experience of other workers who have failed to isolate any specific microorganism from the bones or skin lesions of affected patients. $^{2}$

Another unusual feature of our patient was the symmetrical and erosive nature of the arthropathy; although about $30 \%$ of patients with the syndrome may develop arthritis, it is usually non-erosive and of the mono- or oligoarthritis type. ${ }^{2}$ A rheumatoid-like distribution may occur, as in our patient, but joint erosions are rare, except at the sternoclavicular and sacroiliac joints. ${ }^{2} 5$ The distribution of arthritis in our patient and lack of association with the HLA-B27 antigen in this and other reports ${ }^{2}$ 
raise doubts about the uniform nature of the syndrome and whether indeed it should be classified as one of the seronegative spondyloarthropathies. ${ }^{2}$ Nonetheless, it should be acknowledged that in many patients the syndrome does present with features consistent with a spondarthritis such as sacroiliitis, syndesmophyte formation, and a rash resembling pustular psoriasis. ${ }^{23}$ Our patient does illustrate, however, that arthro-osteitis must also be considered as a possible diagnosis in patients with a seronegative rheumatoid-type polyarthritis. The other unusual featuressclerodactyly and Raynaud's syndrome-were not accompanied by other evidence of connective tissue disease either clinically or by autoantibody testing. At present the significance of these abnormalities and their relation with the arthro-osteitis remains unclear.

The diagnosis was initially overlooked in our patient probably owing to the atypical presentation with the leg predominantly affected, failure of the patient to give a history of palmoplantar pustulosis at the initial presentation, and the apparent rarity of the condition in the United Kingdom population. ${ }^{10}$ Although our awareness of arthro-osteitis was heightened by this patient, no further cases of the disease have been seen on review of 120 consecutive patients attending our seronegative spondylo- arthropathy clinic. Nevertheless, our observations indicate that arthro-osteitis may easily be missed unless clinical suspicion is high and should be considered in all patients who present with sclerotic bone lesions, even in sites outside the sternocostoclavicular region.

We thank Dr B F Boyce for reviewing the bone histology.

1 Sonozaki H, Azuma A, Okai K, et al. Clinical features of 23 cases with inter-sterno-costo-clavicular ossification-a new cases with inter-sterno-costo-clavicular ossification-a new
rheumatic syndrome. Archives of Orthopaedic and Traumatic Surgery 1979; 95: 13-22.

2 Sonozaki $\mathbf{H}$, Mitsui $\mathbf{H}$, Miyanaga $Y$, et al. Clinical features of 53 cases with pustulotic arthro-osteitis. Ann Rheum Dis 1981; 40: 547-53

3 Sozonaki $\mathrm{H}$, Kawashima $\mathrm{M}$, Hongo $\mathrm{O}$, et al. Incidence of arthro-osteitis in patients with pustulosis palmaris et plantaris. Ann Rheum Dis 1981; 40: 554-7.

4 Chigira $M$, Maehara $S$, Nagase $M$, Ogimi $T$, Udagawa $E$, Gunma M. F Bone foint Surg [Am] 1986; 68: 103-12.

5 Edlund E, Johnsson U, Lidgren L, et al. Palmoplantar pustulosis and sternocostoclavicular arthro-osteitis. Ann
Rherum Dis 1988; 47: 809-15.

6 Seppala J, Forssman L, Hypen M. Scand J Rheumatol 1987; 16: 135-8.

7 Kohler H, Uelinger E, Kutzner J, West T B. Sternocostoclavicular hyperostosis: painful swelling of the sternum, clavicles and upper ribs. Ann Intern Med 1977; 87: 192-4.

8 Resnick D, Vint V, Poteshman N L. Sternocostoclavicular hyperostosis. F Bone Joint Surg [Am] 1981; 63: 1329-32.

$9 \mathrm{Kahn} M \mathrm{~F}$, Chamot A M, Benhamou C L, et al. The osteoarticular syndrome acne pustulosis hyperostosis osteitis
(SAPHO) [Abstract]. Br $\mathcal{H}$ R heumatol 1987,26 (suppl): 99 .

10 Sloan R L, Kemmet D, Merrick M V, Nuki G. Arthroosteitis is a rare complication of palmo-plantar pustulosis in a UK population [Abstract]. Br $\mathcal{F}$ Rheumatol 1989; 28 (suppl): 18. 